\title{
Mycotic Thoracoabdominal Aortic Aneurysm Caused by Salmonella cholerasuis
}

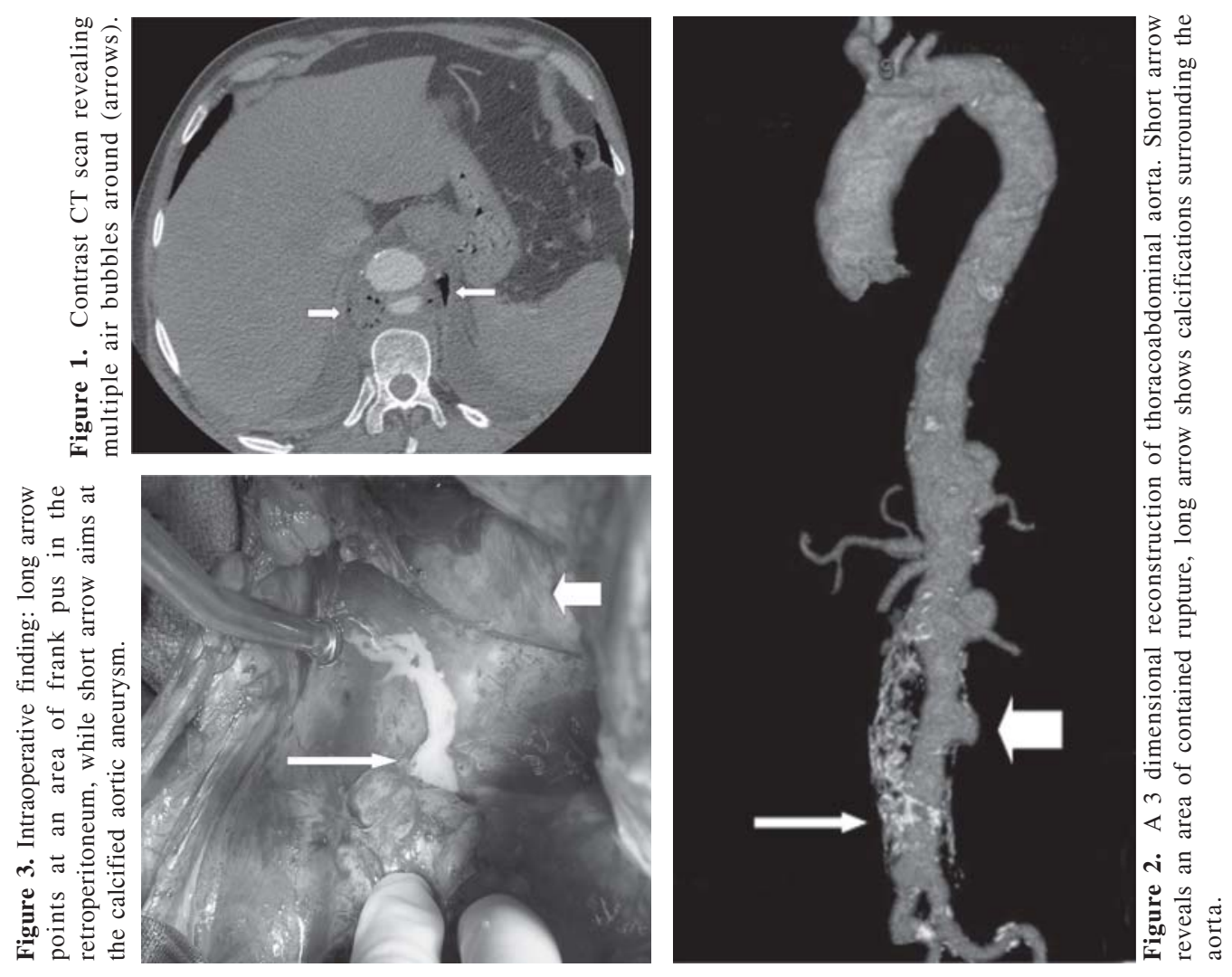

A 69-year-old Caucasian male with history of hypertension, diabetes, and coronary artery disease was admitted to a local hospital for diarrhea and dehydration. It was thought that his symptoms were secondary to food poisoning and the patient was discharged 24 hours later after intravenous fluid administration. Three weeks later the patient came to our hospital with mid abdominal pain radiating to his back. The pain was dull, persistent and moderate in intensity. On examination, the patient was febrile and tachycardic. His abdomen was mildly distended, and tender to deep palpation in the mid abdominal area. There was no rebound or guarding. The results of laboratory tests revealed a WBC count of 18,300 cells $/ \mathrm{mm}^{3}$ with a differential cell count of $82 \%$ neutrophils, $9 \%$ lymphocytes and $9 \%$ monocytes. No bands were seen. His hemoglobin, platelet count, chemistry panel and liver function tests did not reveal frank abnormalities. The CT scan of the chest, abdomen and pelvis (Figure 1) showed a type four thoracoabdominal aortic aneurysm (Crawford classification) of $4.9 \mathrm{~cm}$ of diameter with multiple areas of contained aortic rupture (Figure 2). The aneurysm was surrounded by an inflammatory mass with gas, representing a mycotic aneurysm. The infrarenal segment of the aorta extending to the iliac bifurcation was filled with mural thrombus. The patient's blood cultures grew Salmonella cholerasuis only resistant to penicillin. Treatment with ceftriaxone and ciprofloxacin was initiated promptly, and followed by a complex operation for the mycotic aneurysm. Upon entering the retroperitoneal portion of the aorta, a large area of frank purulent collection was encountered (Figure 3).

Postoperatively, the patient developed acute renal failure. On postoperative day \#14, he suffered a massive episode of aspiration pneumonitis, and succumbed from hypoxemia on the same day.

Ziba Jalali $^{1}$, Miguel Madariaga ${ }^{1}$ and Ali Khoynezhad ${ }^{2}$

${ }^{1}$ University of Nebraska Medical Center Department of Internal Medicine, Section of Infectious Diseases; ${ }^{2}$ University of Nebraska Medical Center, Department of Surgery, Section of Cardiothoracic surgery; Omaha, Nebraska; USA

E-mail: zjalali@unmc.edu 